\title{
Entanglement Entropy after Double Excitation as an Interaction Measure
}

\author{
Yuya Kusuki॰* \\ Center for Gravitational Physics, Yukawa Institute for Theoretical Physics (YITP), Kyoto University, \\ Kitashirakawa Oiwakecho, Sakyo-ku, Kyoto 606-8502, Japan \\ Masamichi Miyajiఠ \\ Department of Physics, Faculty of Science, University of Tokyo, Tokyo 113-0033, Japan \\ and Center for Theoretical Physics and Department of Physics, University of California, Berkeley, California 94720, USA
}

(Received 29 August 2019; revised manuscript received 29 December 2019; accepted 22 January 2020; published 13 February 2020)

\begin{abstract}
We study entanglement entropy after a double local quench in two-dimensional conformal field theories (CFTs). In the holographic CFT, such a state with double excitation is dual to an anti-de Sitter space with two massive particles injected from the boundary. We show that the growth after the double local excitations in pure CFT is universal and is given by the sum of two local quenches with an additional negative term. This negative contribution can be interpreted naturally in holography as being due to the attractive force of gravity. On the CFT side, this evaluation of the entanglement entropy is accomplished by a special limit of six-point functions, where we employ the fusion matrix approach for multipoint conformal blocks developed by the authors [J. High Energy Phys. 08 (2019) 063].
\end{abstract}

DOI: 10.1103/PhysRevLett.124.061601

Introduction.-The AdS/CFT correspondence (holographic principle) [1] has provided new insights in quantum gravity since we understand conformal field theories (CFTs) relatively well. It is expected that there will be holographic duals of quantum gravity theories in three-dimensional antide Sitter space $\left(\mathrm{AdS}_{3}\right)$ (i.e., holographic CFTs), and, in general, they are expected to be unitary, compact CFTs with central charge $c>1$ and without chiral primaries (hence, with no extra currents apart from the Virasoro current), which we call pure CFTs (see, e.g., Refs. [2-4]).

One interesting question is how the dynamics of a twoparticle state in AdS is realized on the CFT side. In particular, we make an effort to understand the dynamics in AdS by making use of the entanglement entropy, which is one very useful tool to access the global structure of a given quantum field theory $[5,6]$. The entanglement entropy is defined by

$$
S_{A}=-\operatorname{tr} \rho_{A} \log \rho_{A},
$$

where $\rho_{A}$ is a reduced density matrix for a subsystem $A$, obtained by tracing out its complement. In practice, this quantity is calculated as the $n \rightarrow 1$ limit of the Renyi entropy, which is a generalization of the entanglement entropy as

Published by the American Physical Society under the terms of the Creative Commons Attribution 4.0 International license. Further distribution of this work must maintain attribution to the author(s) and the published article's title, journal citation, and DOI. Funded by SCOAP .

$$
S_{A}^{(n)}=\frac{1}{1-n} \log \operatorname{tr} \rho_{A}^{n} .
$$

Our interest is the dynamics of the entanglement, which has been developed in 2D CFTs [7-10], and for our purposes, we particularly consider a locally excited state $|\Psi\rangle$, which is defined by acting with a local operator $O(-l)$ on the CFT vacuum $|0\rangle$ in the following manner,

$$
|\Psi(t)\rangle=\sqrt{\mathcal{N}} \mathrm{e}^{-\epsilon H-i H t} O(-l)|0\rangle,
$$

where $\mathcal{N}$ is the normalization factor and an infinitesimal positive parameter $\epsilon$ is an ultraviolet regularization. We choose the subsystem $A$ to be the half-space $x>0$ and apply the excitation in its complement. The growth of the entanglement attributed to the excitation can be seen from the difference between the excited state and the ground state,

$$
\Delta S_{A}^{(n)}[O](t)=S_{A}^{(n)}(|\Psi(t)\rangle)-S_{A}^{(n)}(|0\rangle),
$$

which is our main interest in this Letter.

In CFT, this quantity can be calculated in terms of a correlator [11,12], and it has already been studied in rational conformal field theories (RCFTs) $[13,14]$ and holographic CFTs [15-17], and also nonperturbatively in pure CFTs [4,18,19]. (See also Refs. [20-25], which revealed the growth of the entanglement entropy after a local quench in other setups.) It is interesting to note that the entanglement growth in RCFTs approaches constant in the late time limit, on the other hand, the entanglement entropy in holographic CFTs shows a logarithmic growth. 
This difference is thought to be due to the chaotic nature of holographic CFTs. In other words, we expect that this late time behavior can also be used as a criterion of chaotic nature of a given quantum field theory.

One may wonder what happens if we consider a locally double-excited state instead of a single-excited state,

$$
|\Psi(t)\rangle=\sqrt{\mathcal{N}} \mathrm{e}^{-\epsilon H-i H t} O_{A}\left(-l_{A}\right) \mathrm{e}^{\epsilon H+i H t} \mathrm{e}^{-\epsilon H-i H t} O_{B}\left(-l_{B}\right)|0\rangle .
$$

In this case, one needs to consider higher point correlation functions. Note that higher point functions were evaluated in Refs. [26-28] in a special limit; however, this does not work in our case for the reason discussed in Refs. [4,19,29]. In this Letter, we employ the fusion kernel method, which enables us to approach this problem.

At least in RCFTs, it is naturally expected that the entanglement growth for this state can be given by just a sum rule as simple as

$$
\Delta S_{A}\left[O_{A} ; O_{B}\right]=\Delta S_{A}\left[O_{A}\right]+\Delta S_{A}\left[O_{B}\right],
$$

where $\Delta S_{A}$ is the growth of the entanglement entropy after a double excitation with $O_{A}$ and $O_{B}$. This is because the entanglement entropy in RCFTs behaves as if correlations were carried by free quasiparticles [30]. Actually this rule was already shown in Refs. [14,23,31].

However, there is no reason that the sum rule could also be applied to other CFTs. In particular, the sum rule is unlikely to be true is holographic CFTs, since the quasiparticle picture breaks down in holographic CFTs. For this reason, it would instead be natural for the sum rule not to work in holographic CFTs. The gravity dual of the excited state (3) is obtained by a heavy falling particle in AdS [16]; therefore, the double-excited state (5) should correspond to two falling particles. Since these two particles interact with each other by the gravity force, we expect that the effect of this interaction would also appear in the entanglement growth $\Delta S_{A}\left[O_{A} ; O_{B}\right]$; that is, it is not given by just the sum rule.

With this background, the main aim of this Letter is to verify whether the entanglement entropy measures the interaction between two excitations. This question has remained unexplored because we have little knowledge of the Regge singularity of a correlator, which is necessary to calculate the Renyi entropy. Recently, we investigated this singularity numerically in Refs. $[19,29]$ and gave the analytic form in Ref. [4] from our new findings of many properties of the conformal blocks [18,32] (see also Ref. [33]). The key point is that the singularity of a correlator in the Regge limit is controlled by the pole structure of the monodromy matrix. By utilizing this development, we are able to provide the entanglement entropy for a double-excited state.

Single excitation.-In this section, we will give a brief review of the calculations of the Renyi entropy after a single excitation. Here, we consider a local excitation $O$

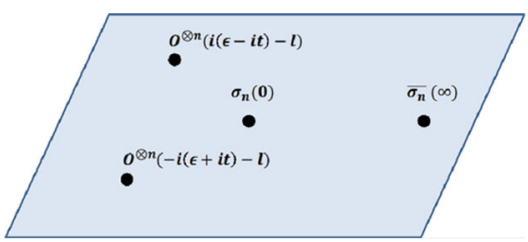

FIG. 1. The holomorphic part of the positions of operators in the replica computation (7).

separated by a distance $l$ from the boundary of $A$, as shown on the left side of Fig. 1. The Renyi entropy in this setup can be calculated using the twist operators $[4,17,19]$

$$
\Delta S_{A}^{(n)}[O]=\frac{1}{1-n} \log \frac{\left\langle\mathbb{O} \mathbb{O} \sigma_{n} \bar{\sigma}_{n}\right\rangle}{\langle\mathbb{O} \mathbb{O}\rangle\left\langle\sigma_{n} \bar{\sigma}_{n}\right\rangle},
$$

where we introduce some notations on the cyclic orbifold CFT $\mathcal{M}^{n} / \mathbb{Z}_{n}$ in Table I. And we also introduce the notations usually found in Liouville CFTs,

$c=1+6 Q^{2}, \quad Q=b+\frac{1}{b}, \quad h_{i}=\alpha_{i}\left(Q-\alpha_{i}\right)$.

By using the cross ratio $z=\left(z_{12} z_{34} / z_{13} z_{24}\right)$, we can rewrite Eq. (7) as

$$
\frac{\left\langle\mathbb{O} D \sigma_{n} \bar{\sigma}_{n}\right\rangle}{\langle\mathbb{O O}\rangle\left\langle\sigma_{n} \bar{\sigma}_{n}\right\rangle}=\left|z^{2 h_{\sigma_{n}}}\right|^{2} G(z, \bar{z}),
$$

where $G(z, \bar{z})$ is the four-point function

$$
G(z, \bar{z})=\left\langle\mathbb{O}(\infty) \mathbb{O}(1) \bar{\sigma}_{n}(z, \bar{z}) \sigma_{n}(0)\right\rangle,
$$

and, in our setup, the cross ratio $(z, \bar{z})$ is given by

$$
z=\frac{2 i \epsilon}{l-t+i \epsilon}, \quad \bar{z}=-\frac{2 i \epsilon}{l+t-i \epsilon} .
$$

TABLE I. The notations on the cyclic orbifold CFT $\mathcal{M}^{n} / \mathbb{Z}_{n}$. We will use these notations in the following.

$\mathcal{F}^{(n)} \quad$ The conformal block associated with the chiral algebra $(\text { Vir })^{n} / \mathbb{Z}_{n}$ instead of the Virasoro. (Note that this conformal block is defined in the CFT with the central charge $n c$.)

$\mathbf{F}^{(n)} \quad$ The fusion matrix associated with the chiral algebra $(\mathrm{Vir})^{n} / \mathbb{Z}_{n}$.

$\mathbf{M}^{(n)} \quad$ The monodromy matrix associated with the chiral algebra $(\text { Vir })^{n} / \mathbb{Z}_{n}$.

$h_{\sigma_{n}} \quad$ The conformal dimension of the twist operators; $h_{\sigma_{n}} \equiv(c / 24)\left[1-\left(1 / n^{2}\right)\right]$.

$\alpha_{n} \quad$ The Liouville momentum of $h_{\sigma_{n}} ; h_{\sigma_{n}} \equiv \alpha_{n}\left(Q-\alpha_{n}\right)$.

(1) The operator defined on the cyclic orbifold CFT $\mathcal{M}^{n} / \mathbb{Z}_{n}$ using the following operators in the seed CFT $\mathcal{M}$ : $O^{\otimes n}=O \otimes O \otimes \cdots \otimes O(\equiv \mathbb{O})$. 
From these expressions, one finds that the sign of the imaginary part of the cross ratio $z$ changes at $t=l$. As a result, the holomorphic cross ratio picks up the factor $\mathrm{e}^{-2 \pi i}$ at $t=l$ as $1-z \rightarrow \mathrm{e}^{-2 \pi i}(1-z)$. On the other hand, this does not happen for the antichiral coordinate $\bar{z}$. Note that the limit $z \rightarrow 0$ after picking up this holomorphic monodromy is usually called the Regge limit.

In general, the four-point function $G(z, \bar{z})$ can be decomposed into conformal blocks as

$$
\sum_{\beta} C_{\beta \sigma_{n} \bar{\sigma}_{n}} C_{\beta \mathbb{O O}^{\dagger}} \mathcal{F}^{(n) \bar{\sigma}_{n} \sigma_{n}}\left(h_{\mathbb{O O}^{\dagger}} \mid z\right) \overline{\mathcal{F}^{(n)^{\left(\sigma_{n} \sigma_{n}^{\dagger}\right.}}}\left(h_{\beta} \mid \bar{z}\right),
$$

where $\beta$ runs over all $(\mathrm{Vir})^{n} / \mathbb{Z}_{n}$ primary operators. The monodromy effect on the conformal block is expressed in terms of the monodromy matrix as [4]

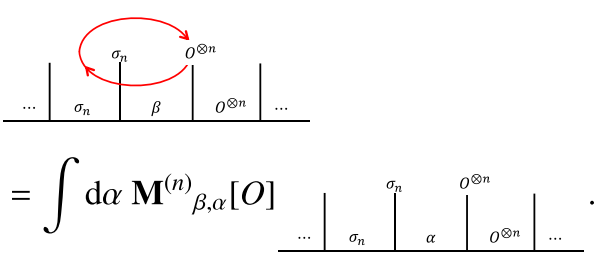

The key point is that if we restrict ourselves to pure CFTs, the minimal value of $\alpha$ is realized by a particular residue at $\alpha=\alpha^{\min }$,

$$
\alpha^{\min }=\min \left\{2 \alpha_{O}, 2 \alpha_{n}\right\} .
$$

From now on, we will focus on the late time region $(t>l)$. In the $\epsilon \rightarrow 0$ limit, the four-point function can be approximated by the vacuum contribution as

$$
\int d \alpha \mathbf{M}_{0, \alpha}^{(n)}[O] \mathcal{F}^{(n)_{\mathrm{OO}^{\dagger}}^{\bar{\sigma}_{n} \sigma_{n}}}\left(n h_{\alpha} \mid z\right) \overline{\mathcal{F}^{(n)^{\bar{\sigma}_{n} \sigma_{n}}}}(0 \mid \bar{z}) .
$$

Here, we used the following asymptotics of the conformal block $\mathcal{F}^{(n)}$,

$$
\mathcal{F}^{(n)_{0_{0}^{+}}^{\bar{\sigma}_{n} \sigma_{n}}}\left(n h_{\alpha} \mid z\right) \underset{z \rightarrow 0}{\longrightarrow} z^{n h_{\alpha}-2 h_{\sigma_{n}}}
$$

This also means that, in the limit $\epsilon \rightarrow 0$, the dominant contribution of the integral over $\alpha$ comes from the minimal one $\alpha=\min \left\{2 \alpha_{O}, 2 \alpha_{n}\right\}$. As a result, the entanglement entropy (the $n \rightarrow 1$ limit of the Renyi entropy) is given by

$\frac{c}{6} \log \frac{t i}{2 \epsilon}+\lim _{n \rightarrow 1} \frac{1}{1-n} \log \left[\operatorname{Res}\left(-2 \pi i \mathbf{M}_{0, \alpha}^{(n)}[O] ; 2 \alpha_{n}\right)\right]$.

And we can also give the $n$th $(n \geq 2)$ Renyi entropy after a local quench $h_{O}<[(c-1) / 32]$ as $\frac{n h_{2 \alpha_{O}}}{1-n} \log \left(\frac{2 \epsilon}{t i}\right)+\frac{1}{1-n} \log \left[\operatorname{Res}\left(-2 \pi i \mathbf{M}_{0, \alpha}^{(n)}[O] ; 2 \alpha_{O}\right)\right]$.

These results are consistent with the results from the light cone limit approach and the gravity dual calculation [4]. Moreover, we can find that in the classical limit $(c \rightarrow \infty)$ the monodromy matrix associated with $(\mathrm{Vir})^{n} / \mathbb{Z}_{n}$ can be expressed in terms of the Bekenstein-Hawking entropy as

$\lim _{n \rightarrow 1} \frac{1}{1-n} \log \left[-2 i \operatorname{Res}\left(-2 \pi i \mathbf{M}_{0, \alpha}^{(n)}[O] ; 2 \alpha_{n}\right)\right] \underset{\substack{c \rightarrow \infty \\ h_{o} \gg c}}{\rightarrow} S_{\mathrm{BH}}(O)$,

where $S_{\mathrm{BH}}(O)$ is given by the Cardy formula,

$$
S_{\mathrm{BH}}(O)=2 \pi \sqrt{\frac{c}{6}\left(h_{O}-\frac{c}{24}\right)} .
$$

Double excitation.-Here, we consider a double-excited state with two operators, $O_{A}\left(-l_{A}\right)$ and $O_{B}\left(-l_{B}\right)\left(l_{A}<l_{B}\right)$. The entanglement entropy for this state can be calculated using the following six-point correlator:

$\Delta S_{A}^{(n)}\left[O_{A} ; O_{B}\right]=\frac{1}{1-n} \log \frac{\left\langle O_{A}^{\otimes n} O_{A}^{\otimes n} O_{B}^{\otimes n} O_{B}^{\otimes n} \sigma_{n} \bar{\sigma}_{n}\right\rangle}{\left\langle O_{A}^{\otimes n} O_{A}^{\otimes n} O_{B}^{\otimes n} O_{B}^{\otimes n}\right\rangle\left\langle\sigma_{n} \bar{\sigma}_{n}\right\rangle}$.

In the late time limit (i.e., the Regge limit), this correlator can also be evaluated in the same way as in the single excitation case. Because the antichiral coordinates of operators undergoes trivial monodromy and goes to zero in the Regge limit, the correlator is approximated by the vacuum sector. In other words, the nonvacuum intermediate states between two $O_{A}$ (or $O_{B}$ ) are suppressed in the Regge limit. Because we are considering pure CFT, this implies that the leading term in the correlator in the Regge limit comes purely from the vacuum exchange conformal block. This allows us to apply the monodromy transformation to the vacuum conformal block in order to evaluate the correlator,

$$
\begin{aligned}
& \left\langle O_{A}^{\otimes n} O_{A}^{\otimes n} O_{B}^{\otimes n} O_{B}^{\otimes n} \sigma_{n} \overline{\sigma_{n}}\right\rangle \underset{\text { Regge limit }}{\longrightarrow} \\
& \int \mathrm{d} \beta_{1} \int \mathrm{d} \beta_{2} \mathbf{M}^{(n)}{ }_{0, \beta_{1}}\left[O_{A}\right] \mathbf{M}^{(n)}{ }_{0, \beta_{2}}\left[O_{B}\right]
\end{aligned}
$$

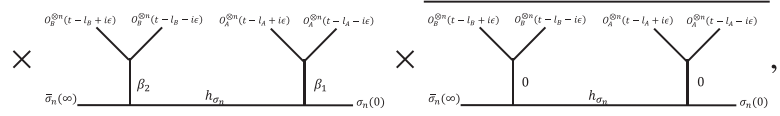

where we used the monodromy transformation (13). Because of Eq. (14), the dominant contributions of the integrals over $\beta_{1}, \beta_{2}$ are given by $\beta_{1}=\beta_{2}=2 \alpha_{n}$, which shows that 


$$
\begin{aligned}
& \frac{\left\langle O_{A}^{\otimes n} O_{A}^{\otimes n} O_{B}^{\otimes n} O_{B}^{\otimes n} \sigma_{n} \bar{\sigma}_{n}\right\rangle}{\left\langle O_{A}^{\otimes n} O_{A}^{\otimes n} O_{B}^{\otimes n} O_{B}^{\otimes n}\right\rangle\left\langle\sigma_{n} \bar{\sigma}_{n}\right\rangle}
\end{aligned}
$$

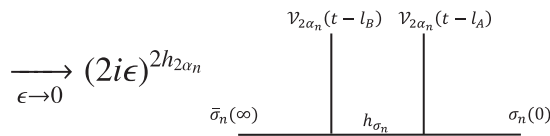

$$
\begin{aligned}
& \times \operatorname{Res}\left(-2 \pi i \mathbf{M}_{0, \alpha}^{(n)}\left[O_{A}\right] ; 2 \alpha_{n}\right) \operatorname{Res}\left(-2 \pi i \mathbf{M}_{0, \alpha}^{(n)}\left[O_{B}\right] ; 2 \alpha_{n}\right) \text {. }
\end{aligned}
$$

Note that the operator $\mathcal{V}_{\alpha}$ indicates a primary with the conformal dimension $h_{\alpha}=\alpha(Q-\alpha)$. The remaining conformal block cannot be evaluated for general $n$; however, if we restrict ourselves to the limit $n \rightarrow 1$, this block has a simple form

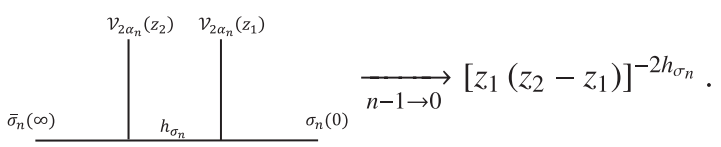

As a result, we obtain

$$
\begin{aligned}
& \frac{\left\langle O_{A}^{\otimes n} O_{A}^{\otimes n} O_{B}^{\otimes n} O_{B}^{\otimes n} \sigma_{n} \bar{\sigma}_{n}\right\rangle}{\left\langle O_{A}^{\otimes n} O_{A}^{\otimes n} O_{B}^{\otimes n} O_{B}^{\otimes n}\right\rangle\left\langle\sigma_{n} \overline{\sigma_{n}}\right\rangle} \\
& \underset{\substack{\epsilon \rightarrow 0 \\
\text { and } \\
n-1 \rightarrow 0}}{\rightarrow}\left[\left(\frac{2 i \epsilon}{t-l_{A}}\right)\left(\frac{2 i \epsilon}{t-l_{B}}\right)\right]^{2 h_{\alpha_{n}}}\left(\frac{l_{B}-l_{A}}{t-l_{A}}\right)^{-2 h_{\sigma_{n}}} \\
& \quad \times \operatorname{Res}\left(-2 \pi i \mathbf{M}_{0, \alpha}^{(n)}\left[O_{A}\right] ; 2 \alpha_{n}\right) \operatorname{Res}\left(-2 \pi i \mathbf{M}_{0, \alpha}^{(n)}\left[O_{B}\right] ; 2 \alpha_{n}\right) .
\end{aligned}
$$

This result leads to the entanglement entropy for the double-excited state in the late time $\left(t>l_{B}\right)$ as

$\Delta S_{A}\left[O_{A} ; O_{B}\right]=\Delta S_{A}\left[O_{A}\right]+\Delta S_{A}\left[O_{B}\right]+\frac{c}{6} \log \frac{l_{B}-l_{A}}{t-l_{A}}$

In summary, although in general two OPE limits of the sixpoint correlator do not give a universal result, the Regge limits in our case give the universal result in the above way. The assumption we used is our CFT is pure; in other words, it does not have chiral currents.

Note that we can remove the restriction that the interval $A$ is large. If we choose the region $A$ to be the interval $[0, L]$ with $L>l_{B}$, then the result of Eq. (26) when $L+l_{B}>$ $t>l_{B}$ becomes

$$
\begin{aligned}
& \Delta S_{A}\left[O_{A} ; O_{B}\right] \\
& \quad=\Delta S_{A}\left[O_{A}\right]+\Delta S_{A}\left[O_{B}\right]+\frac{c}{6} \log \frac{\left(l_{B}-l_{A}\right) L}{\left(t-l_{A}\right)\left[L-\left(t-l_{B}\right)\right]} .
\end{aligned}
$$

And the generalization to put the CFT on a circle with length $L_{\text {tot }}$ is

$$
\begin{aligned}
& \Delta S_{A}\left[O_{A} ; O_{B}\right] \\
& \quad=\Delta S_{A}\left[O_{A}\right]+\Delta S_{A}\left[O_{B}\right]+\frac{c}{6} \log \frac{\sin \left(\pi \frac{l_{B}-l_{A}}{L_{\mathrm{tot}}}\right) \sin \left(\pi \frac{L}{L_{\mathrm{tot}}}\right)}{\sin \left(\pi \frac{t-l_{A}}{L_{\mathrm{tot}}}\right) \sin \left(\pi \frac{L-\left(t-l_{B}\right)}{L_{\mathrm{tot}}}\right)},
\end{aligned}
$$

which can be obtained by the method developed in Ref. [34].

This is the main result of this Letter. The interesting point is that this entanglement entropy (26) cannot be given by just a simple sum of the entanglement entropies for the single excitation with $O_{A}$ and $O_{B}$. In particular, the time dependence in the late time limit $\left(t \gg \epsilon, l_{A}, l_{B}\right)$ is

$$
\Delta S_{A}\left[O_{A} ; O_{B}\right] \sim \frac{c}{6} \log t
$$

which is contrary to the naive expectation that each single excitation contributes to the entanglement entropy as

$$
\Delta S_{A}\left[O_{A} ; O_{B}\right] \sim 2 \times \frac{c}{6} \log t .
$$

We expect that the appearance of the extra term besides $\Delta S_{A}\left[O_{A}\right]$ and $\Delta S_{A}\left[O_{B}\right]$ is the characteristic of the holographic CFT, and this term can be interpreted as an interaction part of the entanglement entropy. In other words, this part measures the interaction between two particles. If so, this interaction part is expected to be negative because this interaction should be dual to gravitational force between two particles, which is attractive (see Ref. [35]). Since $t>l_{B}>l_{A}$, our interaction part is negative; therefore, our result is perfectly consistent with this expectation. Note that our result does not rely on the large $c$ assumption; in other words, our result is exact in central charge [36].

We emphasize that formula (26) holds only in pure CFTs. The point is that, in pure CFTs, the dominant contribution of the integral over $\alpha$ in Eq. (15) comes from $\alpha=2 \alpha_{n}$; however, this is not the case in other CFTs. In RCFTs, the integral (15) is dominated by the vacuum contribution $\alpha=0$, which leads to

$$
\frac{\left\langle O_{A}^{\otimes n} O_{A}^{\otimes n} O_{B}^{\otimes n} O_{B}^{\otimes n} \sigma_{n} \bar{\sigma}_{n}\right\rangle}{\left\langle O_{A}^{\otimes n} O_{A}^{\otimes n} O_{B}^{\otimes n} O_{B}^{\otimes n}\right\rangle\left\langle\sigma_{n} \bar{\sigma}_{n}\right\rangle} \underset{\epsilon \rightarrow 0}{\rightarrow} \mathbf{M}_{0,0}^{(J, n)}\left[O_{A}\right] \mathbf{M}_{0,0}^{(J, n)}\left[O_{B}\right]
$$

where $\mathbf{M}^{(J, n)}$ is the monodromy matrix associated with $(J \text {; current algebra of seed RCFT })^{n} / \mathbb{Z}_{n}$. In fact, this means that the entanglement entropy after the double excitation is given by the sum rule

$$
\Delta S_{A}\left[O_{A} ; O_{B}\right]=\Delta S_{A}\left[O_{A}\right]+\Delta S_{A}\left[O_{B}\right] .
$$

This statement was already shown in Refs. $[14,23,31]$ in another way. For the same reason, this sum rule also appears in free massless scalar field theories [11]. From these observations, we can expect that the appearance of the 
negative interaction term in the entanglement entropy implies the existence of an Einstein gravity dual.

Discussion.-In this Letter, we studied time evolution of entanglement entropy after the double local operator excitations. We assumed the absence of chiral primaries, which allows us to restrict ourselves to the vacuum exchange. We evaluated the deviation of entanglement entropy of double local excitations from the sum of entanglement entropies of each single local excitation. The deviation was found to be negative, which can be interpreted as the attractive force in dual gravity, as was observed in Ref. [35] for joining and splitting quenches.

We emphasize that our CFT result is quite general; that is, we assumed neither a large $c$ limit nor light excitations, but instead only pure CFT. And the result shows that the correction to the entanglement entropy from the gravitational interaction always exist in double local excitation setup, and the correction does not depend on how heavy these operators are.

We now address possible gravity dual of the double locally quenched state. Let us consider the merger of two particles as a candidate and compute the entanglement entropy. We put two conical defects at the boundary of Poincaré AdS, with the same mass and momentum perpendicular to the boundary. Such geometries in global AdS were constructed in Refs. [37-39]. Then these particles eventually form a black hole with horizon radius $r_{h}$ if they have large enough energy. We consider a subregion $[0, L]$ and the corresponding Ryu-Takayanagi surface. At late time, the Ryu-Takayanagi surface probes the region outside the horizon of the black hole, so the entanglement entropy for the subregion $[0, L]$ at late time $l_{A}<l_{B} \ll t \ll L$ is given by

$$
\begin{aligned}
S_{A}\left[O_{A} ; O_{B}\right] \underset{l_{A}<l_{B} \ll t \ll L}{\rightarrow} & \frac{c}{3} \log \frac{L}{\epsilon}+\frac{c}{6} \log \frac{t}{l_{B}-l_{A}} \\
& +\frac{c}{6} \log \left(\frac{\sinh \left(\pi r_{h}\right)}{r_{h}}\right),
\end{aligned}
$$

where $r_{h}$ is the horizon radius of the black hole. Here, we started with the Bañados-Teitelboim-Zanelli geometry

$$
d s^{2}=-\left(r^{2}-r_{h}^{2}\right) d \tau^{2}+\frac{d r^{2}}{r^{2}-r_{h}^{2}}+r^{2} d \phi^{2},
$$

with identification of two surfaces, which are given by

$$
\sqrt{1-\frac{r_{h}^{2}}{r^{2}}} \cosh \left(r_{h} t\right)=\cosh \left(r_{h} \phi\right) \pm \tanh \frac{\pi r_{h}}{2} \sinh \left(r_{h} t\right)
$$

for massless particles. The spacetime region surrounded by these two surfaces is now removed from the spacetime after identification. The intersections of these surfaces at $\phi=0$, $\pi$ correspond to the conical defects. We boosted this geometry so that the spacetime has Minkowski boundary via coordinate transformation

$$
\begin{aligned}
\sqrt{1+r^{2}} \cos \tau & =\frac{\mathrm{e}^{\beta}+\mathrm{e}^{-\beta}\left(z^{2}+x^{2}-t^{2}\right)}{2 z}, \\
\sqrt{1+r^{2}} \sin \tau & =\frac{t}{z}, \\
r \sin \phi & =\frac{x}{z}, \\
r \cos \phi & =-\frac{-\mathrm{e}^{\beta}+\mathrm{e}^{-\beta}\left(z^{2}+x^{2}-t^{2}\right)}{2 z},
\end{aligned}
$$

where $\mathrm{e}^{\beta}=\frac{l_{B}-l_{A}}{2}$. It is interesting to see whether the black hole merger described above or its variants can reproduce the entanglement entropy (26) of double local quench. Indeed, Eqs. (26) and (33) both grow in the late time as

$$
S_{A}\left[O_{A} ; O_{B}\right] \sim \frac{c}{6} \log t .
$$

This behavior can be naturally understood by noting that a two-particle system in the bulk with gravitation should look like a one-particle system at late time. On the other hand, the remaining terms which are time independent depend on the precise dynamics in the bulk, and it seems as if the merger does not reproduce the result of double local quench. Another candidate of gravity dual is given by two massive particles moving in parallel, without a merger that creates a new massive object. It would be very interesting to explore the gravity dual of a double local quench more quantitively and find the dual which can reproduce Eq. (26) correctly.

Finally, we discuss future directions. It would be interesting to consider more refined tools of analysis than the single interval entanglement entropy. For example, the reflected entropy [40] was recently calculated in a single local quench state [41]. This analysis can be generalized to a double local quench state, which would provide more information about the connection between CFT and gravity. It would be interesting to consider other bipartite measures, such as negativity [42,43] and odd entanglement entropy [44]. Further, if we extend our analysis to a multiexcitation of more than 2, then it would also be interesting to study multipartite entanglement or correlation measures (e.g., the generalization of the reflected entropy based on Refs. [45,46]).

We would like to thank Pawel Caputa, Tadashi Takayanagi, and Kotaro Tamaoka for the fruitful discussions and comments. Y. K. and M. M. are supported by the JSPS Fellowship. We are grateful to the conference Quantum Information and String Theory 2019 at the YITP.

*yuya.kusuki@yukawa.kyoto-u.ac.jp masamichimiyaji@berkeley.edu

[1] J. M. Maldacena, in Proceedings of the NATO Advanced Study Institute on Progress in String Theory and M-Theory, Cargese, France, 1999, edited by L. Baulieu, M. Picco, 
P. Windey, and M. B. Green (Springer, New York, 1999), pp. 47-116.

[2] S. Collier, Y.-H. Lin, and X. Yin, arXiv:1608.06241.

[3] S. Collier, P. Kravchuk, Y.-H. Lin, and X. Yin, J. High Energy Phys. 09 (2018) 150.

[4] Y. Kusuki and M. Miyaji, J. High Energy Phys. 08 (2019) 063.

[5] S. Ryu and T. Takayanagi, J. High Energy Phys. 08 (2006) 045.

[6] V. E. Hubeny, M. Rangamani, and T. Takayanagi, J. High Energy Phys. 07 (2007) 062.

[7] P. Calabrese and J. Cardy, J. Stat. Mech. (2007) P10004.

[8] P. Calabrese and J. Cardy, J. Stat. Mech. (2005) P04010.

[9] P. Calabrese and J. Cardy, J. Stat. Mech. (2016) 064003.

[10] P. Calabrese and J. Cardy, Phys. Rev. Lett. 96, 136801 (2006).

[11] M. Nozaki, J. High Energy Phys. 10 (2014) 147.

[12] M. Nozaki, T. Numasawa, and T. Takayanagi, Phys. Rev. Lett. 112, 111602 (2014).

[13] S. He, T. Numasawa, T. Takayanagi, and K. Watanabe, Phys. Rev. D 90, 041701(R) (2014).

[14] T. Numasawa, J. High Energy Phys. 12 (2016) 061.

[15] T. Hartman, arXiv:1303.6955.

[16] P. Caputa, M. Nozaki, and T. Takayanagi, Prog. Theor. Exp. Phys. 2014, 093 B06 (2014).

[17] C. T. Asplund, A. Bernamonti, F. Galli, and T. Hartman, J. High Energy Phys. 02 (2015) 171.

[18] Y. Kusuki, J. High Energy Phys. 01 (2019) 025.

[19] Y. Kusuki and T. Takayanagi, J. High Energy Phys. 01 (2018) 115.

[20] J. R. David, S. Khetrapal, and S. P. Kumar, J. High Energy Phys. 08 (2016) 127.

[21] P. Caputa, Y. Kusuki, T. Takayanagi, and K. Watanabe, J. Phys. A 50, 244001 (2017).

[22] S. He, Phys. Rev. D 99, 026005 (2019).

[23] W.-z. Guo, S. He, and Z.-X. Luo, J. High Energy Phys. 05 (2018) 154.

[24] T. Shimaji, T. Takayanagi, and Z. Wei, J. High Energy Phys. 03 (2019) 165.

[25] L. Apolo, S. He, W. Song, J. Xu, and J. Zheng, J. High Energy Phys. 04 (2019) 009.

[26] T. Anous, T. Hartman, A. Rovai, and J. Sonner, J. High Energy Phys. 07 (2016) 123.
[27] P. Banerjee, S. Datta, and R. Sinha, J. High Energy Phys. 05 (2016) 127.

[28] T. Anous and J. Sonner, Sci. Post. Phys. 7, 003 (2019).

[29] Y. Kusuki, J. High Energy Phys. 07 (2018) 010.

[30] C. T. Asplund, A. Bernamonti, F. Galli, and T. Hartman, J. High Energy Phys. 09 (2015) 110.

[31] P. Caputa and M. M. Rams, J. Phys. A 50, 055002 (2017).

[32] Y. Kusuki, J. High Energy Phys. 08 (2018) 161.

[33] S. Collier, Y. Gobeil, H. Maxfield, and E. Perlmutter, J. High Energy Phys. 05 (2019) 212.

[34] P. Calabrese and J. Cardy, J. Stat. Mech. (2004) P06002.

[35] P. Caputa, T. Numasawa, T. Shimaji, T. Takayanagi, and Z. Wei, J. High Energy Phys. 09 (2019) 018.

[36] One may wonder why the result is universal even in finite $c$. The reason is given in Ref. [4], but here we give a simpler explanation. The contributions from the nonvacuum intermediate states are given by the form $(\epsilon / t)^{\#}$ with a positive nonzero constant \# even in the limit $n \rightarrow 1$ because a state in the twisted sector (in particular, the vacuum state in it) cannot propagate in the OPE between two heavy operators. Thus, they have nonzero dimensions $h, \bar{h}>0$ (due to the compactness of our CFT). On the other hand, the vacuum contribution goes to 1 in the $n \rightarrow 1$ limit. Therefore, the dominant contribution in the von Neumann limit is given only by the vacuum. Thus, the growth shows the universal behavior. Indeed, our procedure perfectly reproduces the previous results for two largely different classes, holographic CFT and RCFT.

[37] H.-J. Matschull, Classical Quantum Gravity 16, 1069 (1999).

[38] D. S. Ageev and I. Y. Aref'eva, Teor. Mat. Fiz. 189, 389 (2016) [Theor. Math. Phys. 189, 1742 (2016)].

[39] I. Y. Aref'eva, M. A. Khramtsov, and M. D. Tikhanovskaya, J. High Energy Phys. 09 (2017) 115.

[40] S. Dutta and T. Faulkner, arXiv:1905.00577.

[41] Y. Kusuki and K. Tamaoka, arXiv:1907.06646.

[42] J. Kudler-Flam and S. Ryu, Phys. Rev. D 99, 106014 (2019).

[43] Y. Kusuki, J. Kudler-Flam, and S. Ryu, Phys. Rev. Lett. 123, 131603 (2019).

[44] K. Tamaoka, Phys. Rev. Lett. 122, 141601 (2019).

[45] K. Umemoto and Y. Zhou, J. High Energy Phys. 10 (2018) 152.

[46] N. Bao and N. Cheng, J. High Energy Phys. 10 (2019) 102. 